\title{
MAGNETO-VISCOELASTIC PLANE WAVES IN ROTATING MEDIA IN THE GENERALIZED THERMOELASTICITY II
}

\author{
S. K. ROY CHOUDHURI AND MANIDIPA BANERJEE (CHATTOPADHYAY)
}

Received 23 June 2004

\begin{abstract}
A study is made of the propagation of time-harmonic magneto-thermoviscoelastic plane waves in a homogeneous electrically conducting viscoelastic medium of Kelvin-Voigt type permeated by a primary uniform external magnetic field when the entire medium rotates with a uniform angular velocity. The generalized thermoelasticity theory of type II (Green and Naghdi model) is used to study the propagation of waves. A more general dispersion equation for coupled waves is derived to ascertain the effects of rotation, finite thermal wave speed of GN theory, viscoelastic parameters and the external magnetic field on the phase velocity, the attenuation coefficient, and the specific energy loss of the waves. Limiting cases for low and high frequencies are also studied. In absence of rotation, external magnetic field, and viscoelasticity, the general dispersion equation reduces to the dispersion equation for coupled thermal dilatational waves in generalized thermoelasticity II (GN model), not considered before. It reveals that the coupled thermal dilatational waves in generalized thermoelasticity II are unattenuated and nondispersive in contrast to the thermoelastic waves in classical coupled thermoelasticity (Chadwick (1960)) which suffer both attenuation and dispersion.
\end{abstract}

\section{Introduction}

The study of propagation of thermoelastic and magneto-thermoelastic waves in nonrotating media was made by several authors. Based on Fourier's law, Biot [2] derived the equations of thermoelasticity which are concerned with the interaction of the thermal field and elastic deformation such that the two fields are coupled. Biot's equations have been used for the investigation of the plane thermoelastic waves. The main drawbacks of Biot's equations are that they were based on Fourier's law which predicts an infinite speed of propagation of heat. Lord and Shulman [16] employed a modified version of Fourier's law to eliminate this paradox and thereby established the generalized coupled heat conduction equation which is hyperbolic in nature. They have derived the equations of dynamic thermoelasticity based on the modified Fourier's law and these equations are usually regarded as the basis of generalized thermoelasticity. Lord and Shulman's equations 
have been used by several authors including Puri [21], Nayfeh and Nemat-Nasser [17] to study the plane thermoelastic waves in an unbounded isotropic homogeneous elastic medium. Agarwal [1] has made an investigation of surface waves in generalized thermoelasticity.

Paria [19] and Wilson [24] investigated the propagation of magneto-thermoelastic waves in a nonrotating medium. These studies, based on the theory of classical coupled thermoelasticity, were essentially concerned with the interaction of the electromagnetic field, the thermal field, and the elastic field, as well as the dispersion relation. In a paper by Schoenberg and Censor [22], the propagation of plane harmonic waves in a rotating elastic medium has been investigated in some details. It has been shown that the rotation causes the elastic medium to be dispersive and anisotropic. This study included some discussion on the free-surface phenomenon in a rotating half-space. Results concerning slowness surfaces, energy flux, reflected waves, and generalized Rayleigh waves have been obtained.

It seems relevant from the above discussion that little attention has been given to the study of propagation of thermoelastic plane waves in a rotating medium in presence of an external magnetic field based on the generalized thermoelasticity. In view of the fact that most large bodies like the earth, the moon, and other planets have an angular velocity, it is important to consider the propagation of magneto-thermoelastic plane waves in an electrically conducting, rotating viscoelastic medium under the action of an external magnetic field. In this connection, Choudhuri and Debnath $[9,10,11,12,13]$ have studied propagation of magneto-thermoelastic plane waves in rotating thermoelastic media permeated by a primary uniform magnetic field using the generalized heat conduction equation of Lord and Shulman. In the present problem, we have studied the propagation of time-harmonic coupled electromagneto-viscoelastic dilatational thermal shear waves using the thermoelasticity theory of type II [14, Green-Naghdi model]. This thermoelastic model possesses several significant characteristics that differ from the traditional classical development in thermoelastic material behaviors: (i) it does not involve thermal energy dissipation, (ii) the entropy flux vector (or equivalently, the heat-flow vector) in the theory is determined in terms of the same potential that also determines the stresses, (iii) it permits transmission of heat flow as thermal waves at finite speed. Several problems in thermoelasticity of type II (without thermal energy dissipation) have been studied by several authors $[4,5,6,7,8,23]$. In this paper, GN model of thermoelasticity of type II is used to obtain a more general dispersion equation to ascertain the effects of rotation, finite thermal waves speed $c_{T}$ of GN theory, thermoelastic coupling constant and the external magnetic field and the viscoelastic parameters on the phase velocity, and attenuation factor of the coupled electromagneto-thermoviscoelastic dilatational shear waves. Special attention is paid to investigate the effects on the specific energy loss for both low and high frequencies. Though several problems of coupled wave propagation have been studied in generalized magneto-thermo-viscoelasticity with/without thermal relaxation by previous researchers, it is believed that this particular problem of coupled wave propagation in a rotating viscoelastic medium in presence of thermal and external magnetic fields using the theory of thermoelasticity of type II (GN model) [14] has not been dealt with before. 


\section{Formulation of the problem and the basic equations}

We consider an infinite, homogenous, isotropic, thermally and electrically conducting viscoelastic solid permeated by a primary magnetic field $\overrightarrow{B_{0}}=\left(B_{1}, B_{2}, B_{3}\right)$. The viscoelastic medium is characterized by the density $\rho$, Lame's constants $\lambda, \mu$, and viscoelastic parameters $\lambda^{\prime}, \mu^{\prime}$, and is uniformly rotating with an angular velocity $\vec{\Omega}=\Omega \vec{w}$, where $\vec{w}$ is the unit vector representing the direction of the axis of rotation. The displacement equation of motion in the rotating frame of reference has two additional terms: centripetal acceleration $\vec{\Omega} \times(\vec{\Omega} \times \vec{u})$ due to the time-varying motion only and the Coriolis acceleration $2 \cdot \vec{\Omega} \times \overrightarrow{\vec{u}}$, where $\vec{u}$ is the dynamic displacement vector. These terms do not appear in a nonrotating medium. The dynamic displacement vector is actually measured from a steady-state deformed position and the deformation is assumed to be small. The displacement equations of motion in a viscoelastic solid of Kelvin-Voigt type with increase of temperature $\theta$ above the reference temperature $\theta_{0}$ are

$$
\begin{aligned}
& \mu \nabla^{2} \vec{u}+\mu^{\prime} \frac{\partial}{\partial t} \nabla^{2} \vec{u}+(\lambda+\mu) \vec{\nabla}(\vec{\nabla} \cdot \vec{u})+\left(\lambda^{\prime}+\mu^{\prime}\right) \frac{\partial}{\partial t} \vec{\nabla}(\vec{\nabla} \cdot \vec{u})+\vec{J} \times \vec{B}-v \vec{\nabla} \theta \\
& \quad=\rho[\ddot{\vec{u}}+\vec{\Omega} \times(\vec{\Omega} \times \vec{u})+2 \vec{\Omega} \times \dot{\vec{u}}],
\end{aligned}
$$

where $\vec{J} \times \vec{B}$ is the electromagnetic body force, $\vec{J}$ is the current density, $\vec{B}=\overrightarrow{B_{0}}+\vec{b}$ is the total magnetic field, $\vec{b}=\left(b_{x}, b_{y}, b_{z}\right)$ is the perturbed magnetic field which is assumed to be small so that the products with $\vec{b}$ and $\vec{u}$ and their derivatives can be neglected for linearization of the field equations, $v=(3 \lambda+2 \mu) \alpha_{0}, \alpha_{0}$ is the coefficient of linear thermal expansion of the solid, and the dots represent the derivatives with respect to time $t$.

The coupled heat conduction equation of the theory of thermoelasticity (type II) without energy dissipation proposed by Green and Naghdi [14] is

$$
\rho c_{v} \ddot{\theta}+v T_{0} \ddot{\Delta}=\rho Q+k^{*} \nabla^{2} \theta
$$

where $c_{v}$ is the specific heat of the solid at constant volume, $\rho$ is the density of the medium, $T_{0}$ is the initial reference temperature, $k^{*}(>0)$ is a material constant characteristic of the theory, $Q$ is the external rate of heat supply per unit mass, and $\Delta$ is the dilatation so that $\Delta=\operatorname{div} \vec{u}$. The finite thermal wave speed is $\left(k^{*} / \rho c_{v}\right)^{1 / 2}$.

In the present problem $Q=0$, so that the heat conduction equation becomes

$$
\rho c_{v} \ddot{\theta}+v T_{0} \ddot{\Delta}=k^{*} \nabla^{2} \theta
$$

Equation (2.3) permits thermal wave propagation without damping. Equations (2.1) and (2.3) are to be supplemented by generalized Ohm's law in a continuous medium with Maxwell's electromagnetic field equations. 
The electromagnetic field is governed by Maxwell's equations with the displacement current and charge density neglected [5]

$$
\begin{gathered}
\vec{\nabla} \times \vec{H}=\vec{J}, \\
\vec{\nabla} \times \vec{E}=-\frac{\partial B}{\partial t}, \\
\vec{\nabla} \cdot \vec{B}=0,
\end{gathered}
$$

where $\vec{B}=\mu_{e} \vec{H}$ and $\mu_{e}$ is the magnetic permeability.

The generalized Ohm's law is

$$
\vec{J}=\sigma\left[\vec{E}+\left(\frac{\partial \vec{u}}{\partial t}+\vec{\Omega} \times \vec{u}\right) \times \vec{B}\right]
$$

where the time-independent part of $\vec{\Omega} \times \vec{u}$ is neglected, $\sigma$ is the electrical conductivity, $\partial \vec{u} / \partial t$ is the particle velocity of the medium, and the small effect of temperature gradient on $\vec{J}$ is also ignored.

\section{Plane wave solutions and dispersion relation}

We consider the propagation of plane waves in the rotating medium in the $x$-direction so that all quantities are proportional to $\exp [i(k x-\omega t)]$, where $(\omega / 2 \pi)$ is the wave frequency and $(2 \pi / k)$ is the wave length. We will assume that $\omega$ is real, but $k$ may be complex. The analysis will be carried out without any discussion of the time-independent stresses and displacements that are caused by the centrifugal force and other possible body forces. We look for time-varying dynamic solutions, and as such, the time-independent part of the centripetal acceleration as well as all body forces will be neglected. However, the time-dependent part of the electromagnetic body force will be taken into consideration. In view of the above assumptions, we write all the field quantities in the form

$$
\begin{aligned}
& \vec{u}=(p, q, r)=\left(p_{0}, q_{0}, r_{0}\right) e^{i(k x-\omega t)}, \\
& T=T_{0} e^{i(k x-\omega t)}, \\
& \vec{J}=\left(j_{1}, j_{2}, j_{3}\right) e^{i(k x-\omega t)}, \\
& \vec{b}=\left(b_{x}, b_{y}, b_{z}\right)=\left(b_{1}, b_{2}, b_{3}\right) e^{i(k x-\omega t)}, \\
& \vec{E}=\left(E_{x}, E_{y}, E_{z}\right), \quad \vec{\Omega}=\left(\Omega_{1}, \Omega_{2}, \Omega_{3}\right),
\end{aligned}
$$

where $p_{0}, q_{0}, r_{0} ; j_{1}, j_{2}, j_{3} ; b_{1}, b_{2}, b_{3} ; \Omega_{1}, \Omega_{2}, \Omega_{3}$, and $T_{0}$ are all constants. 
It follows from (2.4c) that $\operatorname{div} \vec{b}=0$ which implies that $b_{x}=0$, since initially $\vec{b}=\overrightarrow{0}$. Also, it follows from $(2.4 \mathrm{a})$ that $\mu_{e} \vec{J}=\vec{\nabla} \times \vec{b}$ so that

$$
\begin{gathered}
\vec{J}=\left[0,-\frac{i k}{\mu_{e}} b_{z}, \frac{i k}{\mu_{e}} b_{y}\right], \\
\vec{J} \times \overrightarrow{B_{0}}=\left[-\frac{i k}{\mu_{e}}\left(b_{z} B_{3}+b_{y} B_{2}\right), \frac{i k}{\mu_{e}} b_{y} B_{1}, \frac{i k}{\mu_{e}} b_{z} B_{1}\right] .
\end{gathered}
$$

Thus the term $\vec{J} \times \vec{B}$ in (2.1) can be replaced by $\vec{J} \times \overrightarrow{B_{0}}$ given by (3.7).

Substituting (3.1) and (3.2) into (2.3), we find

$$
\begin{gathered}
\theta_{0}=\alpha p_{0}, \\
\alpha=\frac{i v T_{0} k \omega^{2}}{k^{*} k^{2}-\rho c_{v} \omega^{2}} .
\end{gathered}
$$

The equation $\vec{\nabla} \times \vec{E}=-(\partial \vec{b} / \partial t)$ gives

$$
\vec{E}=\left(E_{x}, E_{y}, E_{z}\right)=\left(E_{x}, \frac{\omega}{k} b_{z},-\frac{\omega}{k} b_{y}\right) .
$$

Replacing $\vec{B}$ by primary magnetic field $\overrightarrow{B_{0}},(2.5)$ takes the form

$$
\vec{J}=\sigma\left[\vec{E}+\left(\frac{\partial u}{\partial t}+\vec{\Omega} \times \vec{u}\right) \times \overrightarrow{B_{0}}\right]
$$

Making use of (3.1) and (3.9) and neglecting the product terms, (3.10) with $\vec{J}=\left(J_{x}, J_{y}, J_{z}\right)$ yields

$$
\begin{aligned}
& J_{x}=\sigma\left[E_{x}-i \omega\left(q B_{3}-r B_{2}\right)+B_{3}\left(p \Omega_{3}-r \Omega_{1}\right)-B_{2}\left(q \Omega_{1}-p \Omega_{2}\right)\right], \\
& J_{y}=\sigma\left[\frac{\omega}{k} b_{z}-i \omega\left(r B_{1}-p B_{3}\right)+B_{1}\left(q \Omega_{1}-p \Omega_{2}\right)-B_{3}\left(r \Omega_{2}-q \Omega_{3}\right)\right], \\
& J_{z}=\sigma\left[-\frac{\omega}{k} b_{y}-i \omega\left(p B_{2}-B_{1} q\right)+B_{2}\left(r \Omega_{2}-q \Omega_{3}\right)-B_{1}\left(p \Omega_{3}-r \Omega_{1}\right)\right] .
\end{aligned}
$$

Eliminating $\vec{J}$ from (3.6) and (3.11), we get

$$
\begin{aligned}
& \sigma\left[E_{x}-i \omega\left(q B_{3}-r B_{2}\right)+B_{3}\left(p \Omega_{3}-r \Omega_{1}\right)-B_{2}\left(q \Omega_{1}-p \Omega_{2}\right)\right]=0, \\
& \sigma\left[\frac{\omega}{k} b_{z}-i \omega\left(r B_{1}-p B_{3}\right)+B_{1}\left(q \Omega_{1}-p \Omega_{2}\right)-B_{3}\left(r \Omega_{2}-q \Omega_{3}\right)\right]=-\frac{i k}{\mu_{e}} b_{z}, \\
& \sigma\left[-\frac{\omega}{k} b_{y}-i \omega\left(p B_{2}-q B_{1}\right)+B_{2}\left(r \Omega_{2}-q \Omega_{3}\right)-B_{1}\left(p \Omega_{3}-r \Omega_{1}\right)\right]=\frac{i k}{\mu_{e}} b_{y} .
\end{aligned}
$$


1824 Magneto-viscoelastic plane waves in rotating media

We next put (3.1), (3.2), (3.3), (3.4), and (3.5) into (2.1) and suppress the factor exp[i(kx $-\omega t)]$ throughout the subsequent discussion to obtain the following equations:

$$
\begin{aligned}
& p_{0}\left[\rho\left(\Omega_{1}^{2}-\Omega^{2}-\omega^{2}\right)+(\lambda+2 \mu) k^{2}-(\lambda+2 \mu) i \omega k^{2}+i v \alpha k\right] \\
& \quad+q_{0}\left[\rho\left(2 i \omega \Omega_{3}^{2}+\Omega_{1}^{2} \Omega_{2}^{2}\right)\right]+r_{0}\left[\rho\left(\Omega_{1}^{2} \Omega_{3}^{2}-2 i \omega \Omega_{2}^{2}\right)\right]+\frac{i k}{\mu_{e}}\left(b_{3} B_{3}+b_{2} B_{2}\right)=0, \\
& p_{0}\left[\rho\left(\Omega_{1}^{2} \Omega_{2}^{2}-2 i \omega \Omega_{3}^{2}\right)\right]+q_{0}\left[\rho\left(\Omega_{2}^{2}-\Omega^{2}-\omega^{2}\right)+\mu k^{2}-\mu^{1} i \omega k^{2}\right] \\
& \quad+r_{0}\left[\rho\left(\Omega_{2}^{2} \Omega_{3}^{2}+2 i \omega \Omega_{1}^{2}\right)\right]-\frac{i k}{\mu_{e}} b_{2} B_{1}=0, \\
& p_{0}\left[\rho\left(\Omega_{1}^{2} \Omega_{3}^{2}+2 i \omega \Omega_{2}^{2}\right)\right]+q_{0}\left[\rho\left(\Omega_{2}^{2} \Omega_{3}^{2}-2 i \omega \Omega_{1}^{2}\right)\right] \\
& \quad+r_{0}\left[\rho\left(\Omega_{3}^{2}-\Omega^{2}-\omega^{2}\right)+\mu k^{2}-\mu^{1} i \omega k^{2}\right]-\frac{i k}{\mu_{e}} b_{3} B_{1}=0 .
\end{aligned}
$$

We next rewrite (3.13) and (3.14) in order to obtain their final forms

$$
\begin{aligned}
& p_{0}\left[\sigma\left(i \omega B_{3}-B_{1} \Omega_{2}\right)\right]+q_{0}\left[\sigma\left(B_{1} \Omega_{1}+B_{3} \Omega_{3}\right)\right]+r_{0}\left[-\sigma\left(i \omega B_{1}+B_{3} \Omega_{2}\right)\right] \\
& +b_{3}\left[\frac{i k}{\mu_{e}}+\frac{\sigma \omega}{k}\right]=0, \\
& p_{0}\left[-\sigma\left(i \omega B_{2}+B_{1} \Omega_{3}\right)\right]+q_{0}\left[\sigma\left(i \omega B_{1}-B_{2} \Omega_{3}\right)\right]+r_{0}\left[\sigma\left(B_{2} \Omega_{2}+B_{1} \Omega_{1}\right)\right] \\
& \quad-b_{2}\left[\frac{i k}{\mu_{e}}+\frac{\sigma \omega}{k}\right]=0 .
\end{aligned}
$$

Equations (3.15), (3.16), (3.17), (3.18), and (3.19) constitute a system of five equations with five unknowns $p_{0}, q_{0}, r_{0}$ and the perturbed quantities $b_{2}, b_{3}$.

Since $\vec{b}=\left(0, b_{y}, b_{z}\right)$ and $\vec{b}$-field is normal to $x$-axis, we then choose the $y$-axis and the $z$-axis such that $\vec{b}$-field is along the $y$-axis. Invoking the additional assumption $\Omega_{1}=\Omega_{2}=$ 0 and $\Omega_{3}=\Omega \neq 0$ and considering that $r_{0} \equiv 0$ provided that $\mu k^{2}-\rho \omega^{2} \neq 0$ (evident from (3.17)) so that $B_{3} \equiv 0$, we set the applied and perturbed magnetic fields as $\left(B_{1}, B_{2}, 0\right)$ and $\left(0, b_{2}, 0\right)$, respectively.

This leads to the following three homogenous equations with three unknowns $p_{0}, q_{0}$, and $b_{2}$ as

$$
\begin{aligned}
& p_{0}\left[-\rho\left(\Omega^{2}+\omega^{2}\right)+(\lambda+2 \mu) k^{2}-\left(\lambda^{\prime}+2 \mu^{\prime}\right) i \omega k^{2}+i v \alpha k\right]+q_{0}[\rho 2 i \omega \rho \Omega]+\frac{i k}{\mu_{e}} B_{2} b_{2}=0 \\
& p_{0}[-2 i \omega \rho \Omega]+q_{0}\left[-\rho\left(\Omega^{2}+\omega^{2}\right)+\mu k^{2}-i \omega \mu^{1} k^{2}\right]-\frac{i k B_{1}}{\mu_{e}} b_{2}=0 \\
& p_{0}\left[-\sigma\left(i \omega B_{2}+B_{1} \Omega\right)\right]+q_{0} \sigma\left(i \omega B_{1}-\Omega B_{2}\right)-b_{2}\left[\frac{i k}{\mu_{e}}+\frac{\sigma \omega}{k}\right]=0 .
\end{aligned}
$$


Elimination of $p_{0}, q_{0}, b_{2}$ gives the dispersion equation

$$
\left|\begin{array}{crr}
-\rho\left(\Omega^{2}+\omega^{2}\right)+(\lambda+2 \mu) k^{2}-\left(\lambda^{1}+2 \mu^{1}\right) i w k^{2}+i v \alpha k, & 2 i \omega \rho \Omega, & \frac{i k B_{2}}{\mu_{e}} \\
-2 i \omega \rho \Omega & -\rho\left(\Omega^{2}+\omega^{2}\right)+\mu k^{2}-i \omega \mu^{\prime} k^{2}, & -\frac{i k B_{1}}{\mu_{e}} \\
\sigma\left(i \omega B_{2}+B_{1} \Omega\right), & \sigma\left(\Omega B_{2}-i \omega B_{1}\right), & \left(\frac{i k}{\mu_{e}}+\frac{\sigma \omega}{k}\right)
\end{array}\right|=0 .
$$

It follows from the dispersion equation that the significant effects of the rotation, viscoelasticity, and the thermal field on the phase velocity $\operatorname{Re}(\omega / k)$ are reflected through the terms involving $\Omega, \lambda^{\prime}$, and $\mu^{\prime}$ and the term containing $\alpha$ through $k^{*}$, characteristic of GN theory.

In order to make further simplication of the dispersion equation, we assume $\overrightarrow{B_{0}}=$ $\left(0, B_{2}, 0\right)$ so that $(3.23)$ becomes

$$
\left|\begin{array}{ccc}
-\rho\left(\omega^{2}+\Omega^{2}\right)+(\lambda+2 \mu) k^{2}-\left(\lambda^{\prime}+2 \mu^{\prime}\right) i \omega k^{2}+i v \alpha k, & 2 i \omega \rho \Omega, & \frac{i k}{\mu_{e}} B_{2} \\
-2 i \omega \rho \Omega & -\rho\left(\Omega^{2}+\omega^{2}\right)+\mu k^{2}-i \omega \mu^{\prime} k^{2} & 0 \\
\sigma i \omega B_{2} & \sigma \Omega B_{2}, & \left(\frac{i k}{\mu_{e}}+\frac{\sigma \omega}{k}\right)
\end{array}\right|=0 .
$$

Expanding this determinant and substituting $(\Omega / \omega)=\Omega_{0}$ and the value of $\alpha$ from (3.8b), we obtain

$$
\begin{aligned}
& {\left[\left\{-\frac{\omega^{2}\left(1+\Omega_{0}^{2}\right)}{c_{1}^{2}}+k^{2}-\frac{c_{1}^{2}}{c_{1}^{2}} i \omega k^{2}\right\}\left(k^{*} k^{2}-\rho c_{v} \omega^{2}\right)-\frac{v^{2} k^{2} T_{0} \omega^{2}}{\rho c_{1}^{2}}\right]} \\
& \quad \times\left\{-\rho \omega^{2}\left(1+\Omega_{0}^{2}\right)+\mu k^{2}-i \omega \mu^{\prime} k^{2}\right\}\left(\frac{i k}{\mu_{e}}+\frac{\sigma \omega}{k}\right)+\left(k^{*} k^{2}-\rho c_{v} \omega^{2}\right) \\
& \quad \times\left[-\frac{4 \omega^{2} \rho^{2} \Omega^{2}}{c_{1}^{2}}\left(\frac{i k}{\mu_{e}}+\frac{\sigma \omega}{k}\right)+\frac{k \sigma \omega B_{2}^{2}}{\rho c_{1}^{2} \mu_{e}}\left\{2 \rho \Omega^{2}-\rho \omega^{2}\left(1+\Omega_{0}^{2}\right)+\mu k^{2}-i \omega \mu^{\prime} k^{2}\right\}\right]=0,
\end{aligned}
$$

where $c_{1}=\sqrt{(\lambda+2 \mu) / \rho}$ and $c_{1}^{\prime}=\sqrt{\left(\lambda^{\prime}+2 \mu^{\prime}\right) / \rho}, c_{2}=\sqrt{\mu / \rho}$ and $c_{2}^{\prime}=\sqrt{\mu^{\prime} / \rho}$, where $c_{1}$ is the longitudinal elastic wave velocity and $c_{2}$ is the transverse elastic wave velocity.

It is convenient to introduce the dimensionless quantities

$$
\begin{gathered}
\chi=\frac{\omega}{\omega^{*}}, \quad \xi=\frac{k c_{1}}{\omega^{*}}, \quad \epsilon_{T}=\frac{T_{0} v^{2}}{\rho^{2} c_{v} c_{1}^{2}}, \quad \epsilon_{H}=\frac{\omega^{*} v_{H}}{c_{1}^{2}}, \quad \gamma_{H}=\left(\mu_{e} \cdot \sigma\right)^{-1}, \\
\frac{k^{*}}{\rho c_{v} c_{1}^{2}}=\frac{k^{*} / \rho c_{v}}{c_{1}^{2}}=c_{T}^{2},
\end{gathered}
$$


where $\epsilon_{T}$ is the thermoelastic coupling constant, $c_{T}$ is the nondimensional thermal wave speed of GN theory, depending on $k^{*}, \omega^{*}$ is some standard frequency, and $v_{H}$ is the magnetic viscosity.

For further simplication of (3.25), we observe the results

$$
\begin{aligned}
-\frac{\omega^{2}}{c_{1}^{2}}\left(\Omega_{0}^{2}+1\right)+k^{2}-\frac{c_{1}^{\prime 2}}{c_{1}^{2}} i \omega k^{2} & =\frac{\omega^{*^{2}}}{c_{1}^{2}}\left\{\xi^{2}\left(1-i s_{1}^{2} \chi\right)-\chi^{2}\left(1+\Omega_{0}^{2}\right)\right\}, \\
k^{*} k^{2}-\rho c_{v} \omega^{2} & =k^{*} \frac{\omega^{*^{2}}}{c_{1}^{2}}\left(\xi^{2}-\frac{\chi^{2}}{c_{T}^{2}}\right), \\
\frac{v^{2} k^{2} T_{0} \omega^{2}}{\rho c_{1}^{2}} & =\frac{k^{*} \omega^{*^{4}}}{c_{1}^{4} c_{T}^{2}} \epsilon_{T} \xi^{2} \chi^{2}, \\
-\rho \omega^{2}\left(\Omega_{0}^{2}+1\right)+\mu k^{2}-i \omega \mu^{\prime} k^{2} & =\rho \omega^{*^{2}}\left\{\xi^{2}\left(s^{2}-i \chi s_{2}^{2}\right)-\chi^{2}\left(\Omega_{0}^{2}+1\right)\right\}, \\
\left(\frac{i k}{\mu_{e}}+\frac{\sigma \omega}{k}\right) & =\frac{\sigma c_{1}}{\xi}\left(\chi+i \xi^{2} \epsilon_{H}\right), \\
\frac{4 \omega^{2} \rho \Omega^{2}}{c_{1}^{2}} & =\frac{4 \rho}{c_{1}^{2}} \Omega_{0}^{2} \chi^{4} \omega^{*^{4}}, \\
\frac{k \sigma \omega B_{2}^{2}}{\rho c_{1}^{2} \mu_{e}} & =R_{H} \sigma \xi \chi \frac{\omega^{*^{2}}}{c_{1}} \\
2 \rho \Omega^{2} & =2 \rho \Omega_{0}^{2} \chi^{2} \omega^{*^{2}},
\end{aligned}
$$

where $s_{1}^{2}=\left(c_{1}^{\prime 2} / c_{1}^{2}\right) \omega^{*}, s_{2}^{2}=\left(c_{2}^{\prime 2} / c_{2}^{2}\right) \omega^{*}, s=c_{2} / c_{1}$, and $R_{H}=B_{2}^{2} / \rho c_{1}^{2} \mu_{e}$ is the magnetic pressure number as defined by Pai [18].

Introducing the above result and notations, (3.25) takes the form

$$
\begin{aligned}
& {\left[\left\{\xi^{2}\left(1-i s_{1}^{2} \chi\right)-\chi^{2}\left(\Omega_{0}^{2}+1\right)\right\}\left(\xi^{2} c_{T}^{2}-\chi^{2}\right)-\epsilon_{T} \xi^{2} \chi^{2}\right]\left\{\xi^{2}\left(s^{2}-i \chi s_{2}^{2}\right)-\chi^{2}\left(\Omega_{0}^{2}+1\right)\right\}} \\
& \quad \times\left(\chi+i \xi^{2} \epsilon_{H}\right)+\left(\xi^{2} c_{T}^{2}-\chi^{2}\right)\left[-4 \Omega_{0}^{2} \chi^{4}+R_{H} \xi^{2} \chi\left\{\chi^{2}\left(\Omega_{0}^{2}-1\right)+\xi^{2}\left(s^{2}-i \chi^{2} s_{2}^{2}\right)\right\}\right]=0 .
\end{aligned}
$$

This equation indicates the influence of the rotation and the thermal field through $c_{T}$, $\epsilon_{T}$, and viscoelastic parameters $s_{1}$ and $s_{2}$ on the phase velocity. In the absence of rotation $\left(\Omega_{0}=0\right)$ and viscoelasticity $\left(s_{1}=0, s_{2}=0\right)$ with $R_{L}=B_{1}^{2} / \rho c_{1}^{2} \mu_{e}=0$, the dispersion relation (3.28) reduces to

$$
\begin{gathered}
\left(s^{2} \xi^{2}-\chi^{2}\right)\left[\left(\xi^{2} c_{T}^{2}-\chi^{2}\right)\left(\xi^{2}-\chi^{2}\right)\left(\chi+i \xi^{2} \epsilon_{H}\right)-\chi^{2} \xi^{2} \epsilon_{T}\left(\chi+i \xi^{2} \epsilon_{H}\right)\right. \\
\left.+R_{H} \chi \xi^{2}\left(\xi^{2} c_{T}^{2}-\chi^{2}\right)\right]=0 .
\end{gathered}
$$

In this case, the phase velocity is broken up into two factors. The first factor corresponds to $s^{2} \xi^{2}-\chi^{2}=0$, which leads to a transverse elastic wave (unaffected by thermal field in absence of rotation as expected). 
The other factor leads to

$$
\left(\xi^{2} c_{T}^{2}-\chi^{2}\right)\left[\left\{\xi^{2}-\chi^{2}\right\}\left(\chi+i \xi^{2} \epsilon_{H}\right)+R_{H} \chi \xi^{2}\right]-\epsilon_{T} \chi^{2} \xi^{2}\left(\chi+i \xi^{2} \epsilon_{H}\right)=0
$$

Equation (3.30) corresponds to the dispersion equation of coupled thermal dilatational electrical waves influenced by $k^{*}$ (and hence by $c_{T}$ ), a characteristic of the material of GN model and is not so far dealt with before.

The second factor of (3.29) corresponds to the dispersion equation for coupled thermal dilatational wave influenced by transverse electromagnetic field $R_{H}$.

Setting $R_{H}=0$ in (3.30), the dispersion equation (3.30) reduces to

$$
\left(\chi+i \xi^{2} \epsilon_{H}\right)\left[\left(\xi^{2}-\chi^{2}\right)\left(\xi^{2} c_{T}^{2}-\chi^{2}\right)-\epsilon_{T} \chi^{2} \xi^{2}\right]=0 .
$$

The first factor corresponds to quasistatic oscillations of the electromagnetic field, not coupled with the displacement field, Parkus [20]. The second factor of (3.31) corresponds to dispersion equation (not considered earlier so far) for purely thermoelastic waves (GN model) leading to

$$
\left(\xi^{2}-\chi^{2}\right)\left(\xi^{2} c_{T}^{2}-\chi^{2}\right)-\epsilon_{T} \chi^{2} \xi^{2}=0
$$

in contrast to the equation derived by Chadwick [3] in classical coupled thermoelasticity theory. The roots of this equation are real, indicating that purely thermoelastic waves in thermoelasticity of type II (GN model) are unattenuated (without energy dissipation), not yet considered but are subject to dispersion.

The roots of (3.32) are

$$
\begin{aligned}
& \xi^{2}=\left(M_{1} \pm N_{1}\right) \chi^{2}, \quad \text { where } M_{1}=\frac{1}{2 c_{T}^{2}}\left(c_{T}^{2}+1+\epsilon_{T}\right), \\
& N_{1}=\frac{1}{2 c_{T}^{2}}\left[\left(c_{T}^{2}-1\right)^{2}+\epsilon_{T}^{2}+2 \epsilon_{T}\left(c_{T}^{2}+1\right)\right]^{1 / 2} .
\end{aligned}
$$

The phase speed of the thermoelastic waves in GN model of thermoelasticity is $C_{p}^{E, T}=$ $\chi c_{1} / \xi=c_{1} / \sqrt{\left(M_{1} \pm N_{1}\right)}=V_{E}, V_{T}$ corresponding to $+v e$ and $-v e$ signs.

Setting $\epsilon_{T}=0$ leads to $V_{E}=c_{1}$, (for those materials for which $c_{T}>1$ ) which is the elastic dilatational wave speed and $V_{T}=c_{1} c_{T}=\sqrt{k^{*} / \rho c_{v}}=$ finite thermal wave speed of GN model. Thus $V_{E}$ corresponds to modified elastic dilatational wave speed and $v_{T}$ corresponds to the modified thermal wave speed modified by $c_{T}$ which is the nondimensional thermal wave speed of GN model, a characteristic of the theory and the thermoelastic coupling constant $\epsilon_{T}$. Clearly $v_{E}<v_{T}$, implying that the modified elastic wave follows the modified thermal wave for those materials for which $k^{*}>\rho c_{v} c_{1}{ }^{2}$.

Equation (3.28) represents a more general dispersion relation in the sense that it incorporates the effects of rotation, viscoelasticity and the finite thermal wave speed $c_{T}$, thermoelastic coupling $\epsilon_{T}$, and external magnetic field $R_{H}$. Also, it shows that if the primary magnetic field has a transverse component, the longitudinal and transverse components of the displacement vector are linked together. 
As (3.28) is very complicated, we consider the following limiting cases in order to examine the effects of the rotation, viscoelasticity and the thermal wave speed $c_{T}$, thermoelastic coupling $\epsilon_{T}$, and external magnetic field $R_{H}$ on the phase velocity, on attenuation coefficient of waves, and also on specific energy loss.

\section{Low-frequency region $(\chi \ll 1)$}

In this case, the wave frequency $\omega$ is much smaller than $\omega^{*}$. We consider this case with finite electrical conductivity $\left(\sigma \neq 0, v_{H} \neq 0\right)$. Thus when $\chi=0, \xi^{2}=0$ so that we can write $\xi^{2}=i \phi \chi+0\left(\chi^{2}\right)$, where $\phi$ is to be determined. We substitute $\xi^{2}$ into (3.28), retain the terms containing $\chi^{4}$, and then equate the coefficient of $\chi^{4}$ to zero in order to obtain an equation for $\phi$ as

$$
\phi=\frac{1+R_{H}}{\epsilon_{H}} .
$$

The root of (4.1) corresponds to one kind of slow wave because

$$
\left|\frac{\omega}{k}\right|=\left|\frac{\omega}{\omega^{*}} \frac{\omega^{*}}{k c_{1}} c_{1}\right|=\left|\frac{c_{1} \chi}{\xi}\right| \sim c_{1} 0\left(\chi^{1 / 2}\right) \ll c_{1} .
$$

Thus for the low frequency, the rotation, the thermal field $k^{*}, \epsilon_{T}$, and the viscoelasticity have no influence on the phase velocity in the case of finite conductivity. The equation for $\phi$ is linear so that its only root $\phi=\left(1+R_{H}\right) / \epsilon_{H}$. This corresponds to only one slow wave influenced by the electromagnetic field. This fact was not noticed in the works by Choudhuri and Debnath [12], which reveals two kinds of slow waves. Then the phase velocity can be found from the result

$$
\xi= \pm(1+i)\left(1+\frac{\chi}{2 \epsilon_{H}}\right)^{1 / 2} R_{m}
$$

where $R_{m}^{2}=1+R_{H}=1+v_{A}^{2} / c_{1}^{2}$ and $v_{A}$ is the Alfvèn wave velocity.

It follows from (4.3) that there exists a magneto-elastic wave.

It follows from the real and imaginary parts of $\xi$ that the phase velocity is

$$
c_{p}=c_{1}\left(\frac{\chi \epsilon_{H}}{2}\right)^{1 / 2} R_{m}^{-1} .
$$

The attenuation factor is

$$
a_{f}=\frac{\omega^{*}}{c_{1}}\left(\frac{\chi}{2 \epsilon_{H}}\right)^{1 / 2} R_{m} .
$$

The phase speed and attenuation factor are independent of $c_{T}$, viscoelastic parameters, the thermal wave speed, and thermoelastic coupling $\epsilon_{T}$ to the order of $(\chi)$ for $\chi \ll 1$.

However considering terms of $0\left(\chi^{2}\right)$ for $\chi \ll 1$, we obtain from the general dispersion equation (3.28) that

$$
\xi^{2}=\frac{(A C-B D)+i(A D+B C)}{A^{2}+B^{2}},
$$


where

$$
\begin{aligned}
& A=\epsilon_{H} c_{T}^{2}\left(s^{2}-s_{1}^{2} s^{2} \chi^{2}\right) \\
& B=\epsilon_{H} c_{T}^{2}\left(s_{1}^{2} s^{2}+s_{2}^{2}\right) \chi \\
& C=\chi^{2}\left[\epsilon_{H}\left\{s^{2}\left(1+\epsilon_{T}\right)+c_{T}^{2}\left(\Omega_{0}^{2}+1\right)\left(s^{2}+1\right)\right\}+c_{T}^{2}\left(s_{1}^{2} s^{2}+s_{2}^{2}+R_{H} s_{2}^{2}\right)\right] \\
& D=c_{T}^{2} \chi s^{2}\left(1+R_{H}\right) .
\end{aligned}
$$

It follows from the real and imaginary parts of $\xi$ that the phase velocity is

$$
c_{p}=\frac{\chi c_{1}}{\sqrt{R_{1}}} \cos \frac{\phi}{2}
$$

and the attenuation factor is

$$
a_{f}=\frac{\omega^{*}}{c_{1}} \sqrt{R_{1}} \sin \frac{\phi}{2}
$$

where

$$
\begin{gathered}
R_{1}=\frac{\left[(A C-B D)^{2}+(A D+B C)^{2}\right]^{1 / 2}}{\left(A^{2}+B^{2}\right)} \\
\tan \phi=\frac{A D+B C}{A C-B D}
\end{gathered}
$$

This confirms that the phase speed and the attenuation factors both are affected by rotation, viscoelastic parameters, finite thermal wave speed $c_{T}$, the thermoelastic coupling $\epsilon_{T}$, the external magnetic field, and the electromagnetic parameter $\epsilon_{H}$.

\section{High-frequency region $(\chi \gg 1)$}

This case corresponds to the case of wave frequency $\omega$, much larger than $\omega^{*}$. Dividing the dispersion equation (3.28) by $\chi^{7}$ and neglecting all terms involving the second and higher powers of $(1 / \chi),(3.28)$ becomes

$$
\xi= \pm(1+i)\left[\frac{\chi}{2\left\{\left(\Omega_{0}^{2}+1\right)\left(s_{1}^{2}+s_{2}^{2}\right)+\left(\Omega_{0}^{2}-1\right)^{2} \epsilon_{H}\right\}}\right]^{1 / 2}\left(\Omega_{0}^{2}-1\right) .
$$

Thus the effect of rotation, viscoelastic parameters $s_{1}, s_{2}$, and the electromagnetic parameter $\epsilon_{H}$ on the phase velocity is observed to the first order of $(1 / \chi)$ whereas without viscoelastic effect,

$$
s_{1}=s_{2}=0, \quad \xi= \pm(1+i)\left(\frac{\chi}{2 \epsilon_{H}}\right)^{1 / 2} .
$$

Thus no effect of rotation and thermal parameters $k^{*}, \epsilon_{T}$ on the phase velocity is observed, but however electromagnetic parameter $\epsilon_{H}$ affects it. 
1830 Magneto-viscoelastic plane waves in rotating media

To the first order of $(1 / \chi)$ for $(\chi \gg 1)$, the phase velocity $c_{p}$ and the attenuation coefficient factor $a_{f}$ are as follows:

$$
\begin{gathered}
c_{p}=\frac{c_{1}}{\left(\Omega_{0}^{2}-1\right)}\left[\frac{\chi\left\{\left(\Omega_{0}^{2}+1\right)\left(s_{1}^{2}+s_{2}^{2}\right)+\left(\Omega_{0}^{2}-1\right)^{2} \epsilon_{H}\right\}}{2}\right]^{1 / 2}, \\
a_{f}=\frac{\omega^{*}}{c_{1}}\left[\frac{\chi}{2\left\{\left(\Omega_{0}^{2}+1\right)\left(s_{1}^{2}+s_{2}^{2}\right)+\left(\Omega_{0}^{2}-1\right) \epsilon_{H}\right\}}\right]^{1 / 2}\left(\Omega_{0}^{2}-1\right) .
\end{gathered}
$$

Now dividing (3.28) by $\chi^{7}$, retaining the terms of the order of $(1 / \chi)^{2}$ for $(\chi \gg 1)$ and neglecting the higher powers of $(1 / \chi)$, we obtain

$$
\begin{aligned}
\xi^{4}\left[\frac{s_{1}^{2} s_{2}^{2}}{\chi^{2}}+\right. & \left.\left(\Omega_{0}^{2}+1\right) \frac{s_{2}^{2} \epsilon_{H}}{\chi^{2}}+\left(\Omega_{0}^{2}+1\right) \frac{s_{2}^{2} \epsilon_{H}}{\chi^{2}}\right] \\
+\xi^{2}[ & \left(\Omega_{0}^{2}+1\right) \frac{s^{2}}{\chi^{2}}-i\left(\Omega_{0}^{2}+1\right) \frac{s_{2}^{2}}{\chi}+\frac{\left(\Omega_{0}^{2}+1\right)^{2} c_{T}^{2}}{\chi^{2}}+\frac{\left(\Omega_{0}^{2}+1\right)}{\chi^{2}} \\
& -\left(\Omega_{0}^{2}+1\right) \frac{i s_{1}^{2}}{\chi}+\frac{\left(\Omega_{0}^{2}+1\right)^{2} \epsilon_{T}}{\chi^{2}}-\left(\Omega_{0}^{2}+1\right) \frac{i \epsilon_{H}}{\chi} \\
& \left.-\frac{4 \Omega_{0}^{2} c_{T}^{2}}{\chi^{2}}+\frac{4 \Omega_{0}^{2} i \epsilon_{H}}{\chi}-\frac{R_{H}\left(\Omega_{0}^{2}-1\right)}{\chi^{2}}\right]-\left(\Omega_{0}^{2}-1\right)^{2}=0 .
\end{aligned}
$$

Clearly, the roots of this dispersion equation with complex coefficients are complex, indicating that the coupled magneto-thermo-viscoelastic waves undergo attenuation and dispersion. Both the phase speed and the attenuation coefficients of the coupled waves are influenced by rotation, viscoelastic parameters $s_{1}, s_{2}$, external magnetic field and the finite wave speed $c_{T}$, characteristic of GN theory, and the thermoelastic coupling constant $\epsilon_{T}$ to the second order of $(1 / \chi)$ for large frequency.

In absence of viscoelastic effects, the above equation gives

$$
\xi^{2}=\frac{(L+i M)}{L^{2}+M^{2}}
$$

where

$$
\begin{gathered}
M=\frac{\epsilon_{H}}{\chi}, \\
L=\frac{1}{\chi^{2}\left(\Omega_{0}^{2}-1\right)^{2}}\left[\left(1+\epsilon_{T}+s^{2}\right)\left(\Omega_{0}^{2}+1\right)-R_{H}\left(\Omega_{0}^{2}-1\right)+\left(\Omega_{0}^{2}-1\right)^{2} c_{T}^{2}\right]=\frac{L_{0}}{\chi^{2}},
\end{gathered}
$$


where

$$
L_{0}=\frac{1}{\left(\Omega_{0}^{2}-1\right)^{2}}\left[\left(1+\epsilon_{T}+s^{2}\right)\left(\Omega_{0}^{2}+1\right)-R_{H}\left(\Omega_{0}^{2}-1\right)+\left(\Omega_{0}^{2}-1^{2}\right) c_{T}^{2}\right]
$$

It follows from the real and the imaginary parts of $\xi$ that the phase velocity is

$$
c_{p}=\frac{\chi c_{1}}{\sqrt{R}} \cos \frac{\theta}{2}
$$

and the attenuation factor is

$$
a_{f}=\frac{\omega^{*}}{c_{1}} \sqrt{R} \sin \frac{\theta}{2}
$$

where

$$
R=\frac{1}{\sqrt{L^{2}+M^{2}}}, \quad \tan \theta=\frac{M}{L} .
$$

The results (5.10) and (5.11) are similar to (5.4) and (5.6) reported by Choudhuri and Debnath [12]. The results (5.10) and (5.11) correspond to the phase speed and attenuation factor of the coupled magneto-thermoelastic wave in a rotating medium in thermoelasticity of type II (GN model), not considered so far. These are clearly influenced by $c_{T}$, rotation, $\epsilon_{T}$, and the external magnetic field.

It is important to observe that rotation does exert influence on both the phase velocity and the attenuation factor for high frequencies to the second order of $(1 / \chi)$. Also both the phase speed and the attenuation factor are modified by the applied magnetic field, thermal parameters $k^{*}, \epsilon_{T}$ through the term $L$ for high frequency. This fact was not noticed for the case of low frequency up to the order of $\left(\chi^{2}\right)$.

\section{Specific energy loss}

Making reference to Kolsky [15], the specific energy loss $(\Delta W / W)$ is defined as the ratio of the energy dissipated per stress cycle to the total vibrational energy and is given by

$$
\frac{\Delta W}{W}=\frac{4 \pi}{\omega} c_{p} a_{f}
$$

To the second order of $\chi$ for $\chi \ll 1$, the specific energy loss from (4.8) and (4.9) is given by

$$
\frac{\Delta W}{W}=2 \pi \frac{(A D+B C)}{\left[(A C-B D)^{2}+(A D+B C)^{2}\right]^{1 / 2}} .
$$

Therefore, the specific energy loss is affected by rotation, finite thermal wave speed $c_{T}$, the thermoelastic coupling $\epsilon_{T}$, the external magnetic field, and the electromagnetic parameter. 
To the first order of $1 / \chi$ for $\chi \gg 1$, the specific energy loss is obtained from (5.3) and (5.4) in the form

$$
\frac{\Delta W}{W}=2 \pi
$$

Equation (6.3) shows that the specific energy loss is independent of any field parameters in the case of high frequency up to the first order of $(1 / \chi)$.

However, to the second order of $(1 / \chi)$, the expression for the specific energy loss in absence of viscoelastic effect is obtained from (5.10) and (5.11) in the form

$$
\frac{\Delta W}{W}=2 \pi \frac{M}{\sqrt{L_{0}^{2} / \chi^{4}+M^{2}}} .
$$

This result confirms that the specific energy loss is affected by the rotation to the second order of $(1 / \chi)$ for the case of high frequency and depends on thermal parameters $\epsilon_{T}$, finite thermal wave speed $c_{T}$ of GN theory of thermoelasticity of type II, electromagnetic parameter $\epsilon_{H}$, and the transverse magnetic field.

\section{Discussion}

(1) Magneto-thermo-viscoelastic-dilatational shear waves in generalized thermoelasticity II undergo both attenuation and dispersion in contrast to the purely coupled thermoelastic waves in generalized thermoelasticity II (without thermal energy dissipation) which suffer no attenuation and dispersion.

(2) The coupled waves are influenced by the finite thermal wave speed of GN theory, rotation, viscoelastic parameters, and the external magnetic field, and by the fact that due to the presence of the transverse magnetic field $R_{H}$ and rotation, the longitudinal and transverse motions are linked together.

(3) For low frequency ( $\chi \ll 1, \chi$ being the ratio of the frequency to some standard frequency $\left.\omega^{*}\right)$, the rotation, viscoelastic parameters $s_{1}$ and $s_{2}$, and the thermal field have no effect on the phase velocity to the first order of $\chi$ corresponding to only one slow wave influenced by the electromagnetic field $R_{H}$ only. But to the second order of $\chi$, the phase velocity, attenuation coefficient, and the specific energy loss are affected by rotation, viscoelastic parameters and depend on the finite thermal wave speed $c_{T}$, the thermoelastic coupling $\epsilon_{T}$, electromagnetic parameter, and the transverse magnetic field $R_{H}$.

(4) For large frequency, rotation, viscoelastic parameters, and the electromagnetic parameter $\epsilon_{H}$ influence the phase velocity and attenuation coefficient to the order of $(1 / \chi)$. However, to the order of $(1 / \chi)^{2}$, the phase speed and attenuation coefficients of the waves are affected by rotation, viscoelastic parameters $s_{1}, s_{2}$, the finite thermal wave speed $c_{T}$ of GN theory, the transverse magnetic field $R_{H}$, and the thermoelastic coupling $\epsilon_{T}$.

(5) It reveals also that to the order of $(1 / \chi)$, the specific energy loss is independent of any field parameters and is a constant. Without viscoelastic effect, the specific loss is however affected by rotation, finite thermal wave speed $c_{T}$, external magnetic field $R_{H}$, and the electromagnetic parameter $\epsilon_{H}$ to the second order of $(1 / \chi)$ in the case of magnetothermoelastic waves in rotating media in generalized thermoelasticity II, not studied before. 


\section{References}

[1] V. K. Agarwal, On surface waves in generalized thermoelasticity, J. Elasticity 8 (1978), no. 2, 171-177.

[2] M. A. Biot, Thermoelasticity and irreversible thermodynamics, J. Appl. Mech. Tech. Phys. 27 (1956), 240-253.

[3] P. Chadwick, Thermoelasticity. The dynamical theory, Progress in Solid Mechanics. Vol. 1, North-Holland, Amsterdam, 1960, pp. 263-328.

[4] D. S. Chandrasekharaiah, Thermoelastic plane waves without energy dissipation, Mech. Res. Comm. 23 (1996), no. 5, 549-555.

[5] - A uniqueness theorem in the theory of thermoelasticity without energy dissipation, J. Thermal Stresses 19 (1996), no. 3, 267-272.

[6] D. S. Chandrasekharaiah and K. S. Srinath, Axisymmetric thermoelastic interactions without energy dissipation in an unbounded body with cylindrical cavity, J. Elasticity 46 (1997), no. 1, $19-31$.

[7] _ Thermoelastic plane waves without energy dissipation in a rotating body, Mech. Res. Comm. 24 (1997), no. 5, 551-560.

[8] Thermoelastic interactions without energy dissipation due to a point heat source, J. Elasticity 50 (1998), no. 2, 97-108.

[9] S. K. Roy Choudhuri, Electro-magneto-thermo-elastic plane waves in rotating media with thermal relaxation, Internat. J. Engrg. Sci. 22 (1984), no. 5, 519-530.

[10] - On magneto-thermoelastic plane waves in infinite rotating media with thermal relaxation, Proc. IUTAM Symposium on Electro-Magneto Mechanical Interactions In Deformable Solids and Structure, (Tokyo, Japan) (Y. Yamamoto and K. Miya, eds.), NorthHolland, Amsterdam, 1986, p. 361.

[11] _ Magneto-thermo-micro elastic plane waves in finitely conducting solids with thermal relaxation, Proc. IUTAM Symposium on Mechanical Modelling of new electro-magneticmaterials, (Sweden, Stockholm) (R. K. T. Hsieh, ed.), Elsevier Science Publisher, Amsterdam, 1990, pp. 461-468.

[12] S. K. Roy Choudhuri and L. Debnath, Magneto-thermo-elastic plane waves in rotating media, Internat. J. Engrg. Sci. 21 (1983), no. 2, 155-163.

[13] _ Magnetoelastic plane waves in infinite rotating media, J. Appl. Mech. 50 (1983), 283287.

[14] A. E. Green and P. M. Naghdi, Thermoelasticity without energy dissipation, J. Elasticity 31 (1993), no. 3, 189-208.

[15] H. Kolsky, Stress Waves in Solids, Monographs on the Physics and Chemistry of Materials, vol. 13, Oxford: At the Clarendon Press, London, 1953.

[16] H. W. Lord and Y. Shulman, A generalized dynamical theory of thermoelasticity, J. Mech. Phys. Solids 15 (1967), 299-309.

[17] A. Nayfeh and S. Nemat-Nasser, Thermoelastic waves in solids with thermal relaxation, Acta Mech. 12 (1971), 53-69.

[18] S. I. Pai, Some considerations of the fundamental equations of electro-magneto-gasdynamics, Calcutta Math. Soc. Golden Jubilee Commemoration. Vol. (1958/59), Part I, Calcutta Math. Soc., Calcutta, 1963, pp. 235-248.

[19] G. Paria, On magneto-thermo-elastic plane waves, Math. Proc. Cambridge Philos. Soc. 58 (1962), 527-531.

[20] H. Parkus (ed.), Electro Magnetic Interactions in Elastic Solids, CISM Courses and Lectures, vol. 257, Springer, Vienna, 1979.

[21] P. Puri, Plane waves in generalized thermoelasticity, Internat. J. Engrg. Sci. 11 (1973), 735-744.

[22] M. Schoenberg and D. Censor, Elastic waves in rotating media, Quart. J. Mech. Appl. Math. 31 (1973), 115-125. 
1834 Magneto-viscoelastic plane waves in rotating media

[23] J. N. Sharma and R. N. Chouhan, On the problem of body forces and heat sources in thermoelasticity without energy dissipation, Indian J. Pure Appl. Math. 30 (1999), no. 6, 595-610.

[24] A. J. Wilson, The propagation of magneto-thermoelastic plane waves, Math. Proc. Cambridge Philos. Soc. 59 (1963), 438-488.

S. K. Roy Choudhuri: Department of Mathematics, Burdwan University, Burdwan-713 104, West Bengal, India

E-mail address: skrc_bu_math@yahoo.com

Manidipa Banerjee (Chattopadhyay): Department of Mathematics, Burdwan University, Burdwan -713 104, West Bengal, India 


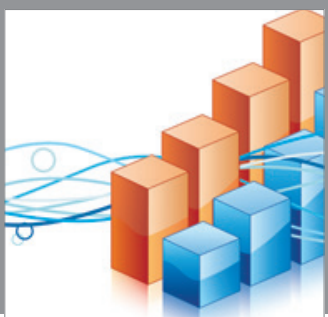

Advances in

Operations Research

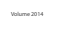

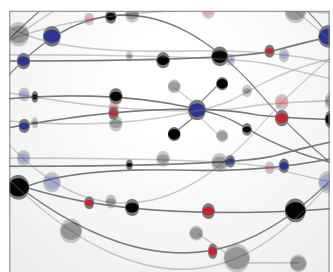

\section{The Scientific} World Journal
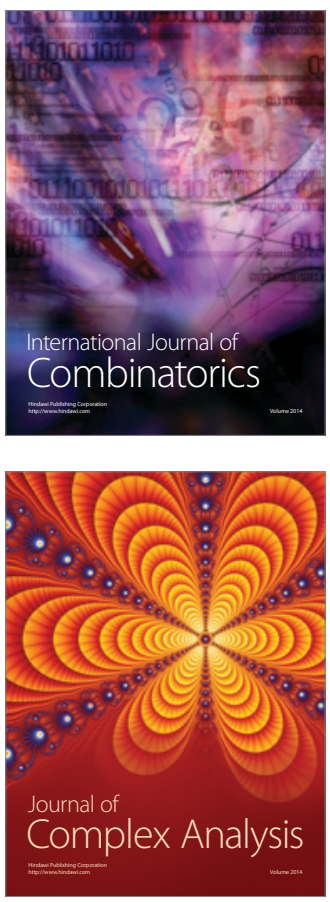

International Journal of

Mathematics and

Mathematical

Sciences
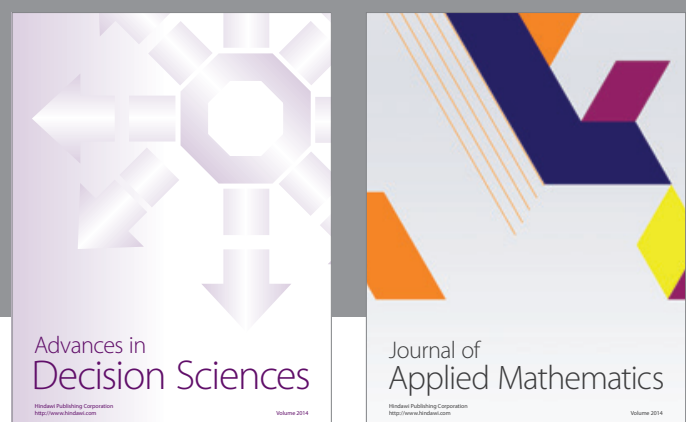

Journal of

Applied Mathematics
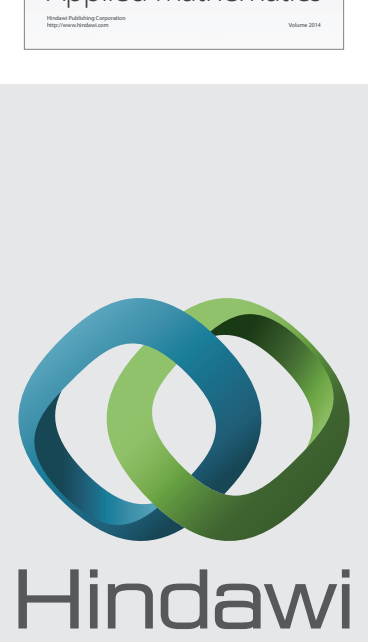

Submit your manuscripts at http://www.hindawi.com
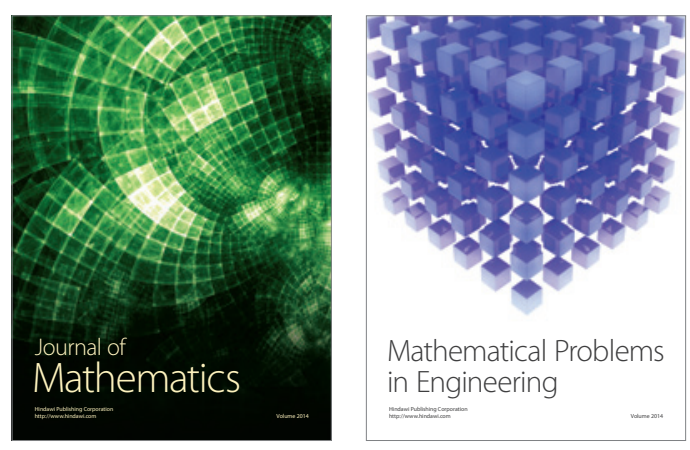

Mathematical Problems in Engineering
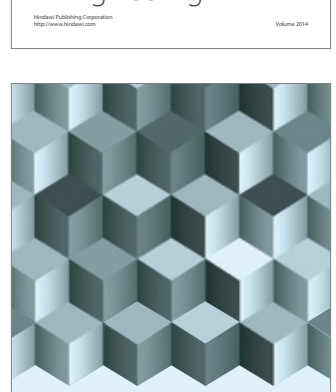

Journal of

Function Spaces
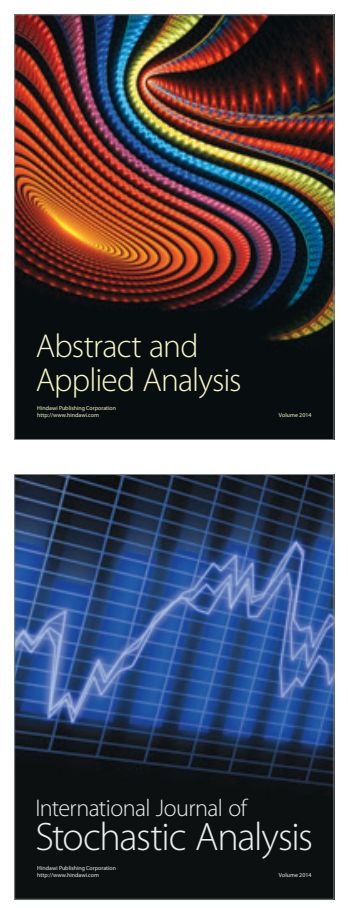

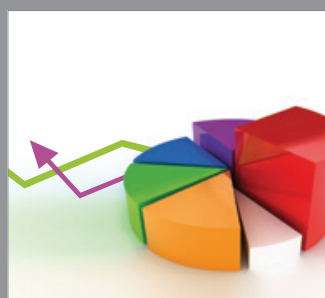

ournal of

Probability and Statistics

Promensencen
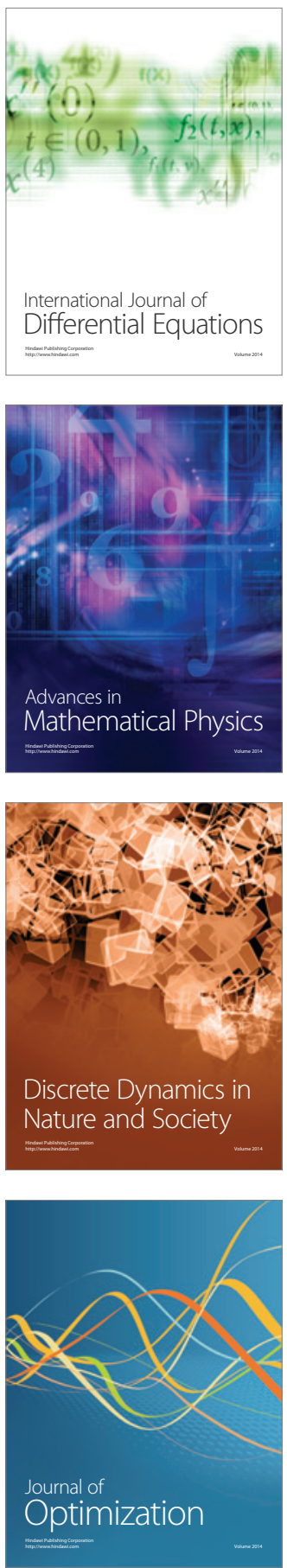